\title{
Design of Drone3dContour: A Novel Contouring System using Altitude Measurement and Cloud-Web Computing
}

\author{
Kantilal P Rane \\ Professor, KCEs COEIT, Jalgaon, India, kantiprane@ rediffmail.com
}

\begin{abstract}
3D modelling of land survey is necessary for various geographical applications like surveying, levelling and geographic analysis. Traditional method for contouring using dumpy level is a measurement of altitude with reference of a reference point that requires manual measurement and provides accuracy of $0.17 \mathrm{~m}$. This method is time consuming to perform the manual process for more number of points of survey. Manual errors may be introduced in this method. The quick altitude measurement and 2D \& 3D contour formation method is required to be developed for accurate analysis. A novel method for altitude measurement is proposed using controlled drone that scans the specified area in predefined manner and measures the corresponding altitudes of drone from the earth surface points to calculate the altitudes of every earth surface point with reference to the initial reference point. The altitude data of the specified earth surface points are send to cloud for storage and computing purpose. Cloud computing is performed for 2D $\& 3$ D contour formation using MATLAB cloud computing functions. Final information is displayed on web site and can be accessed remotely from anywhere through proper authentication.
\end{abstract}

Key words: Drone Control, Altitude measurement, 2D \& 3D contour, Cloud computing, web-server

\section{INTRODUCTION}

Contour lines (isolines) visualize surfaces of continuous levelling information as curved lines that joining all the points of equal altitude. Contour lines are depict lines having constant altitude/elevation and are always used to display various parameters such as air quality, population density, air pressure or weather maps. Land levelling plays major role in modern infrastructural development. It requires the $2 \mathrm{D}$ contour formation and $3 \mathrm{D}$ modelling of land to be surveyed. Information about altitudes corresponding to the locations along specified number of land points is required to create the contour.

From 1950s, there was in use a very tedious procedure and more complicated normal contouring technique [1].
Modern cartographic [2] and GIS software packages [3] were mostly used but they lack in quickly and easily creating customized contour lines. GIS and image-editing software [4] is a time-consuming and multi-step process. Various modern tools like GMT (Generic Mapping Tools) [5], LASTools [6], NCAR Graphics Software Package [7] were developed for contour formation. All these tools require geographic and Cartesian data sets for contour formation. Data manipulation like filtering, trend fitting, gridding, projecting, etc can be performed by open source GMT [5]. It is a collection of tools of 80 basic command lines and 40 more specialized and discipline-specific tools. Through it, it can produce various forms of contours like simple $\mathrm{x}$-y plots by contour maps, artificially illuminated layered surfaces and 3D views. It can also perform over 30 map projections and transformations. Visualization of GSHHG coastlines, political boundaries, and rivers map is also possible using GMT. Along with contouring, LASTools [6] can perform accessing of LIDAR data from and/to compressed LAZ and standard LAS file and can also converts ASCII from/to Shapefiles. Various operations like viewing, filtering, thinning, merging, DEM rasterizing, TIN triangulating, and creation of boundary polygon were possible using this tool. NCAR Graphics software [7] was developed by Fortran and C for different operations like Contour plots, XY plots, Surfaces/isosurfaces maps, Streamline plots, Triangular meshes, Vector plots, Weather maps and Histograms.

Controlled drone based contour formation is a novel approach for surveying purpose. The various controlled drones like Phantum series drone, Pixhawk drone $[8,9]$ are already used for various geo-graphical applications. Various features of various drones $[8,9]$ are needed to be identified for its proper selection in particular application.

We used MATLAB programming for creating and modelling 2D and 3D models of captured altitude data as the altitude information is proposed to be stored in cloud in this application. ThingSpeak cloud [10] is used for storage of extracted altitude information. This information is used by MATLAB programming for computing the required information like 2D contour and 3D model of land mark. The computed 2D and 3D models are then displayed on 
Kantilal P Rane, International Journal of Emerging Trends in Engineering Research, 8(6), June 2020, 2395 - 2401

web page. Web-server [11] using MATLAB function development [12], MATLAB Compiler SDK [13], MATLAB Production Server [14] and The Web Developer Workflow using .NET [12]; is designed for visualizing the extracted final information to website.

\section{METHODOLOGY}

Proposed system includes the various subsystems as, 1) Controlled Drone: It performs the various tasks like two type of altitudes measurement by using inbuilt fly height of the drone and laser distance sensor. It scans the field using pre-defined path to collect the required information 2) Base Station: It remotely accesses all the collected information from drone and performs data transformation and sends it to ThingSpeak cloud, 3) Cloud Computing System: It uses MATLAB programming with cloud data and creates the 2D and 3D contour plot of land mark, 4) Web-server: It accesses the information to be displayed on web site for remote accessing using proper authentication.

Process flow of the proposed system is defined as follows.

1) Initialization of Controlled Drone, Base Station, Cloud System and Web-server.

2) Feed the pre-defined path for scanning of field.

3) Fly the Controlled Drone at pre-defined height (h1). Move it to pre-defined point of scan.

4) Altitude measurement using fly-height of the drone and compare it with h. If not equal, it automatically adjusts the h1 level.

5) Altitude measurement by laser distance sensor attached to drone (A).

6) Scanning the field for remaining pre-defined points of pre-defined path for collecting the information like altitudes (h and A), longitude (L1) and latitude (L2) using steps 3) to 5).

7) Remote accessing of all the collected information of all the scan points from drone to Base Station (Controller cum long range wifi access point).

7) Transform the raw information to applicative information by Base Station and sends it to ThingSpeak cloud for storage.

8) Cloud computing using MATLAB cloud programming with stored data that creates the $2 \mathrm{D}$ contour plot and $3 \mathrm{D}$ model plot of field land mark.

9) Display all the extracted plots and information on website using .NET web-server for remote accessing using proper authentication.

\subsection{Controlled Drone}

The drone including various features like computerized controllability, fly-height real time accessing facility, image capturing facility, additional electronic device carrying and controlling facility; is the major requirement for the proposed system. After survey of various drones, their features and fly-height accuracy [15], DJI Phantom 3 Standard Quad-copter Drone is selected for proposed application. It can be controlled according to the specified points of scan on pre-defined path at specified fly-height. Scan point's longitude, latitude and fly-height altitude (called Input Database) are to be provided to drone system for its proper positioning and it also can be stored/recorded in real time by controlling software and real time database can be easily accessible using the controlling software to interfaced hardware (controller) or software [16]. After positioning to the particular point, it can be programmed for capturing the information like clicked photographs and the actual measured altitude A using external long range sensor attached to it. To capture the photograph, camera is used and affixed under the drone and to capture actual altitude from the earth surface, Long Range Laser Distance Sensor Controller (LRLDSC) is used and is also affixed under it. Both image and height sensing can be clicked (captured) simultaneously using single control action but the captured image and corresponding measured altitude can be sent to base station through different communication media. Captured image may be stored into SD card and measured actual attitude A may be send to base station through wifi communication from LRLDSC to Base Station.

The altitude of earth surface point $\left(\mathrm{C}_{i j}\right)$ is calculated on the basic of reference point height $(\mathrm{H})$ from sea level, flyheight of drone (h) at reference point and measured altitude $\left(\mathrm{A}_{i j}\right)$ by LRLDSC i.e. distance between drone and the earth surface point where $i$ and $j$ indicates the scan line and scan line indices respectively.

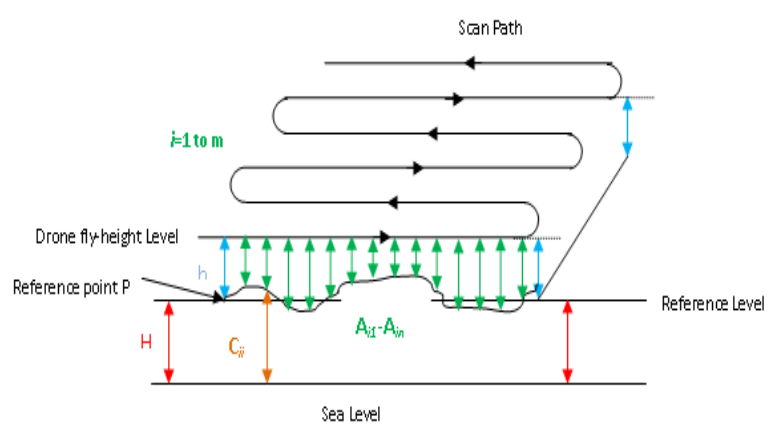

Figure 1: Altitude Estimation Process

As shown in Figure 1, drone is initially started on reference point $\mathrm{P}$ on the earth surface. It is vertically fly for the height $\mathrm{h}$ at point $\mathrm{p}_{11}$ using drones controlled software. Using LRLDSC, altitude of drone from earth surface point is measured using long rang laser distance sensor. At this initial point, measurement is calibrated by comparing flyheight of drone with measured height by LRLDSC. Altitude $\mathrm{A}_{11}$ is then recorded. Drone with same fly-height is moved to point $\mathrm{p} 2$ along scan line (by providing longitude and latitude of $\mathrm{p}_{12}$ ) to capture its corresponding Altitude $\left(\mathrm{A}_{12}\right)$ by LRLDSC. Similarly all the altitudes 
Kantilal P Rane, International Journal of Emerging Trends in Engineering Research, 8(6), June 2020, 2395 - 2401

along $1^{\text {st }}$ scan line are measured and dataset is created as $\mathrm{C}_{1}$. Similar data sets $\mathrm{C}_{2}, \mathrm{C} 3, \ldots, \mathrm{C}_{\mathrm{m}}$ are recorded to create final database $\mathrm{C}$. After reaching to final point $\mathrm{P}_{m n}$, drone is returned to base station on earth surface on point $\mathrm{P}$ and final dataset $\mathrm{C}$ is send to base station to ThingSpeak cloud through base station wifi connection.

Let dataset $\mathrm{C}_{i}$ be the sub-set of altitude of all the scan points along the horizontal scan line of index $i$ and dataset $\mathrm{C}$ is the subset of altitude of all the scan points over all the scan lines.

$\mathrm{C}_{i}=\left\{(\mathrm{H}+\mathrm{h})-\mathrm{A}_{i j}\right\}$ for interval $j=1$ to $\mathrm{n}$ for scan lines $i=1$ to $\mathrm{m}$.

$\mathrm{C}=\left\{\mathrm{C}_{i}\right\}$ for all $i=1$ to $\mathrm{m}$.

Dataset $\mathrm{C}$ is stored to the cloud and can be used to design of contour using cloud computing using MATLAB programming. A MATLAB function called Cloud2D3DContour.m is implemented for transforming the dataset $\mathrm{C}$ into the $2 \mathrm{D}$ contour and $3 \mathrm{D}$ model of land mark.

\subsubsection{LRLDSC}

Long Range Laser Distance Sensor Controller (LRLDSC) is system consisting of Arduino controller interfaced with ESP 8266 wifi module and long range laser distance sensor as shown in Figure 2. When system is initialized, it connects to the wifi access point of base station using ESP8266 wifi module. Input is then taken from drone system to capture the height of earth surface vertically using laser distance sensor. Drone system when reaches to pre-defined position provides capture signal to LRLDSC. Arduino controller identifies the signal and measures the altitude using distance sensor. At initial, it performs the auto-calibration and then from $2^{\text {nd }}$ scan point, it measures the corresponding altitude and temporarily stores in its memory. If no signal is received from drone system in specified time, then it sends all the collected data through wifi ESP 8266 module to base station for further processing.

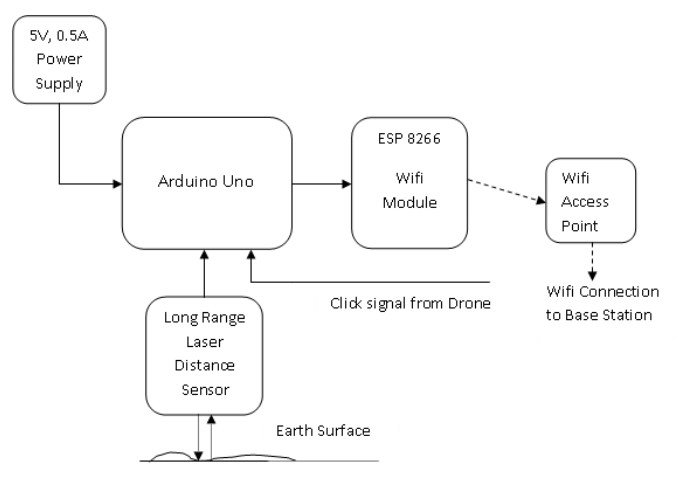

Figure 2: Block schematic for LRLDSC
Algorithmic steps for the LRLDSC embedded C programming is as follows.

1) Initializes the system and ESP 8266 for connection with base station.

2) Scan the input signal for getting signal from Drone controller for starting the altitude capture

3) If signal is not received, wait for scanning the input line signal for next $40 \mathrm{sec}$, else exit.

4) If signal is received, send the capture signal to long range altitude sensor to measure the distance vertically from drone to earth surface in normal direction.

5) Wait for $15 \mathrm{sec}$ for capturing the data from distance sensor.

6) Store the received signal from memory.

7) Repeat the step 2) to 6) until exit.

8) When exit, if new data is stored, sends it to the base station using wifi connection.

9) Repeat the step 2) to step 8).

\subsection{Base Station}

Base station (Figure 3) is a Raspberry Pi controller based system to which wifi module (ralink rt5370 usb wifi adapter) is connected to access data through wifi connection. External long range access point is used for accessing about $100 \mathrm{~m}$ range. Raspberry Pi controller is configured to connect to Arduino Controller through wifi connection via long range wifi access point. Input Database as specified in section 2.1 is also stored in to the memory at pre-defined location of Raspberry Pi controller before initialization of scanning process. After initializing of scanning process, Base Station undergoes to data receiving mode from Arduino wifi connection. After finishing the work of Arduino Controller, it sends the captured data to Base Station. Base station receives it and stores it into the specified memory. It then modifies the Input Database by adding information from Arduino Controller (Altitude of corresponding scan points) into new database called Output Database. This database is then sent to ThingSpeak cloud for storage and for further processing.

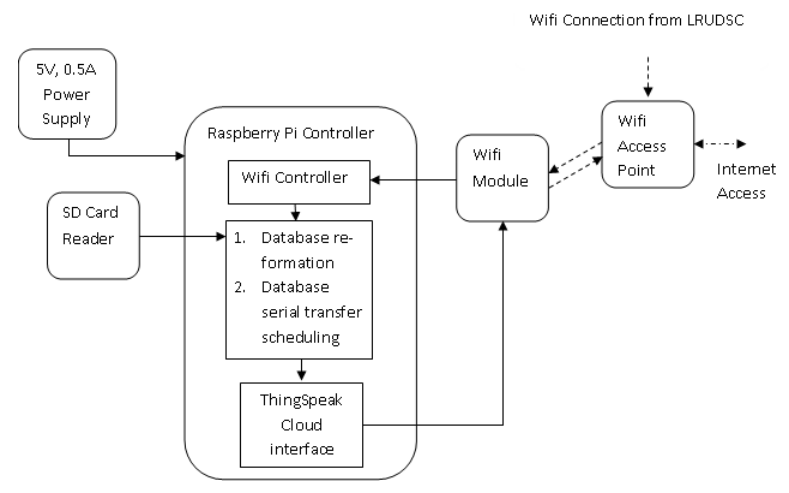

Figure 3: Block schematic for Base Station 
Kantilal P Rane, International Journal of Emerging Trends in Engineering Research, 8(6), June 2020, 2395 - 2401

Algorithmic steps for the Base Station Python programming is as follows.

1) Initialize the wifi module and connection to Arduino Controller using wifi connection.

2) Verify / initialize the Internet connectivity.

3) Initialize the ThingSpeak cloud connection using Api key.

4) Check for serial data from wifi connection.

5) If data is not available then again check for it until data received.

6) Received data is verified, compared and modified with corresponding longitude and latitude field as Output Database.

7) Send data from this database to ThingSpeak cloud one by one for storage and further processing.

\subsection{Cloud Computing System}

MATLAB cloud computing is performed to create 2D and $3 \mathrm{D}$ contour plot of land mark. MATLAB algorithm called Cloud2D3DContour.m is developed that can access the uploaded information (Output Database) when required and perform the plot operations for modelling of 3D view of surveyed field for user visualization and for further analysis. User can execute the MATLAB algorithm (code) for performing cloud computing through .net interface for graphical display and for command processing.

Algorithmic steps of function Cloud2D3DContour.m are as follows.

1) Downloads the Output Database from ThingSpeak cloud.

2) Find the maximum and minimum level of measured altitude field.

3) Divide the altitude range into different levels according to the specified levels by user.

4) Distribute the data set into subset-data sets according to the different levels of altitudes.

5) Convert longitude and latitude information of all the scan points into the $\mathrm{x}-\mathrm{y}$ positions with specific scale (initially defined).

6) Plot the dot point of all the scan points over $x-y$ plot.

7) Connect all the dot points of same subset-data (initially $1^{\text {st }}$ subset-data of $1^{\text {st }}$ level) in curvature form and plot close path.

8) Perform step 7) for all the subset-data and plots corresponding plots.

9) Interpolate the points on curvature to enlarge the subset-data of all the levels called horizontal interpolation.

10) Add the sub-levels between every pair of levels (called parents levels) according to resolution (initially) specified. And interpolate the data according to corresponding altitude levels of points of parent's levels. It increases the number of altitude levels.

11) Plot the data with $3 \mathrm{D}$ plot.

12) Provide the different colours for points of different levels in 3D plot.

13) If provided resolution is not sufficient then it automatically adjusts (increases) the resolution and performs the step 10) to step 12) for proper visualization in Auto-resolution-set mode.

\subsection{Web-server}

ASP.NET web-server [11] is designed for user interface to execute the different commands. These commands can execute the MATLAB algorithm; can display the results on user interface display on website. Website can be accessed from anywhere using proper user name and password. MATLAB function Call to the .NET environment is performed through the steps as

Following are the steps used for deployment of MATLAB function into webpage using .NET server and MATLAB production server.

1) MATLAB function development [12].

2) Enlist all the inputs and outputs to the function, which can be used for the web development.

3) For deployment of function into MATLAB Production Server [14], the function is compiled and tested using SDK [13].

4) Copy the deployed compiled function in to the MATLAB Production Server framework.

5) Call the MATLAB function into the .NET environment [12] through MATLAB Production Server test environment.

6) Call the final compiled version and as necessary, integrate the output of the function in the web site [12].

\section{RESULTS}

Camera click signal is used for capturing the altitude using DJI Phantom 3 Standard Quad-copter Drone (Figure 4) using LRLDSC for measuring the distance through Laser sensor TF03.

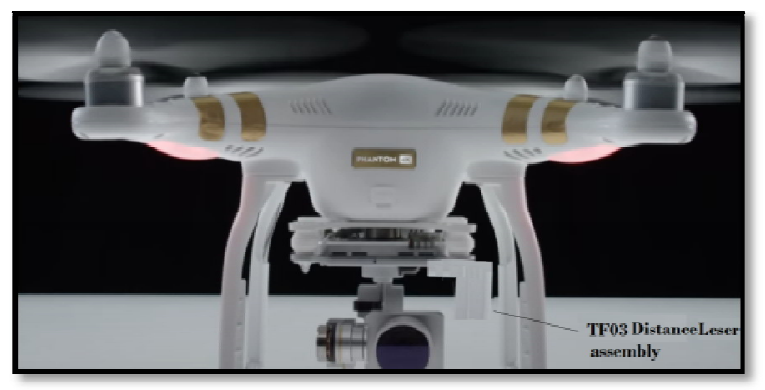

Figure 4: DJI Phantom 3 Standard Quad-copter Drone with LRLDSC Assembly 
Kantilal P Rane, International Journal of Emerging Trends in Engineering Research, 8(6), June 2020, 2395 - 2401

Drone Controlling Software called Dornedeploy is used for scanning the defined surveying field along the pre-defined path as shown in Figure 5. Scan path and altitude graph used is as shown in Figure 5a and Figure 5b respectively.

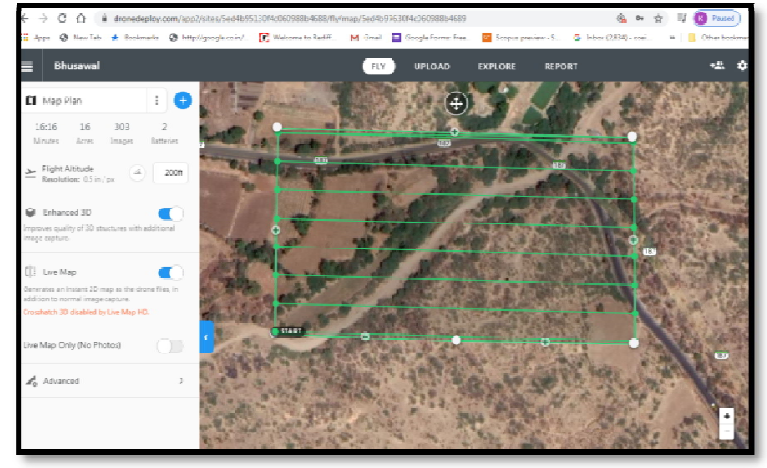

Figure 5a: Drone Controlling Software- scan path

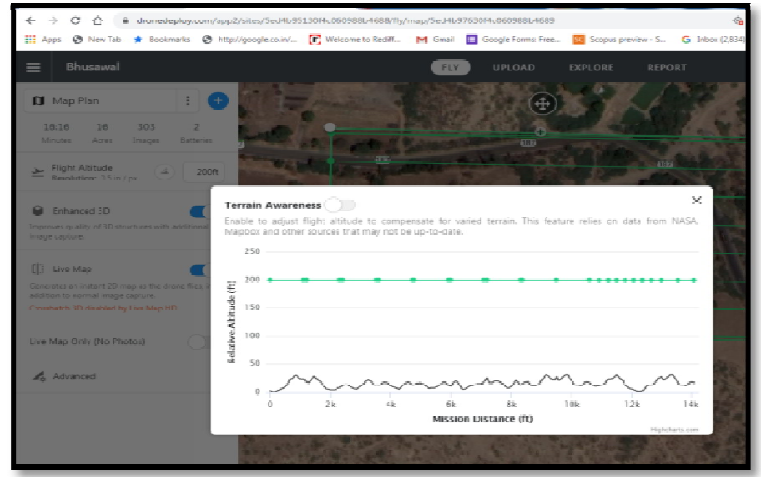

Figure 5b: Drone Controlling Software- attitude Graph

Long distance (100 m range TF03 Lidar sensor) as shown in Figure 6 is used for sensing the earth surface distance from drone. For sensing, LRLDSC is directed normally downward towards the earth surface. Once the drone is stable at pre-defined point, it clicks for measuring the distance from earth surface.

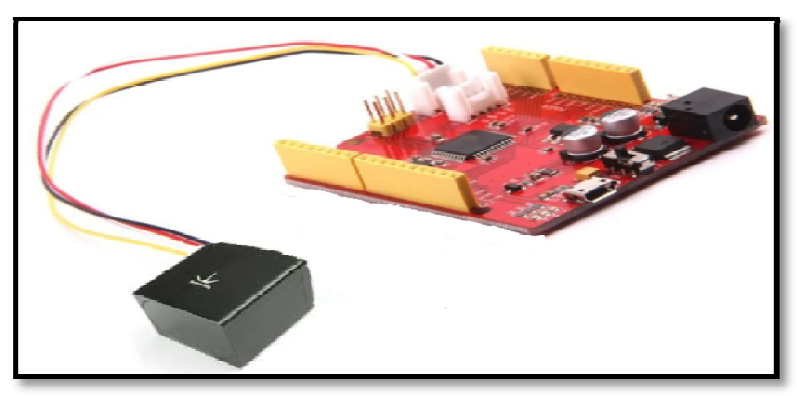

Figure 6: LRLDSC having Long range Laser Distance Sensor TF03

Base Station of used for local wireless communication of captured data after the whole scanning is completed. It includes wifi dongle module and SD card for its operation as shown in Figure 7.

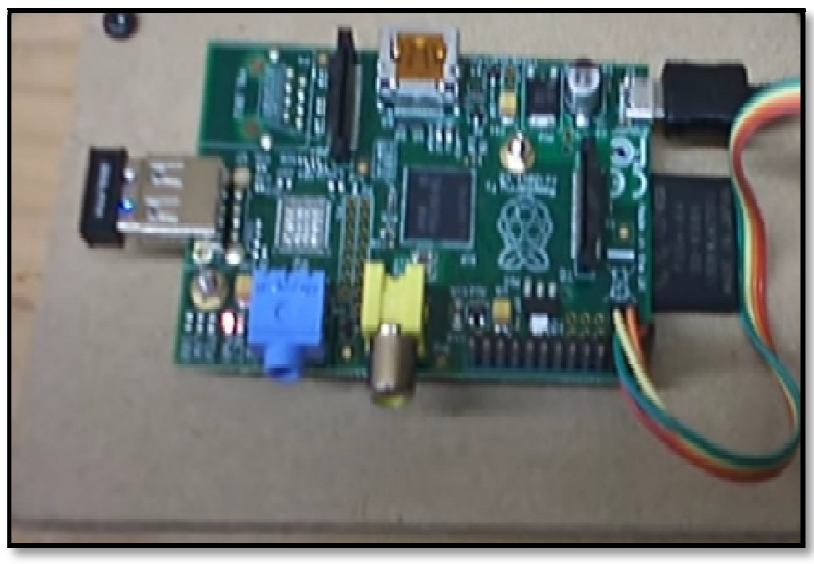

Figure 7: Base Station with Wifi dongle and SD card

ThingSpeak cloud is used for the global storage of captured information. The various distributed databases showing $\mathrm{X}$ position, $\mathrm{Y}$ position and corresponding altitude for few samples are shown in Figure 8a, Figure $8 \mathrm{~b}$ and Figure $8 \mathrm{c}$ respectively.

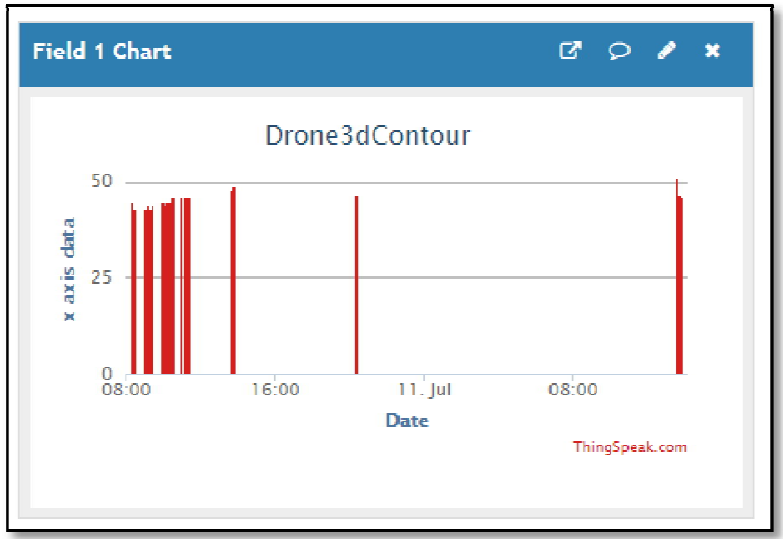

Figure 8a: $\mathrm{X}$ position database from ThingSpeak

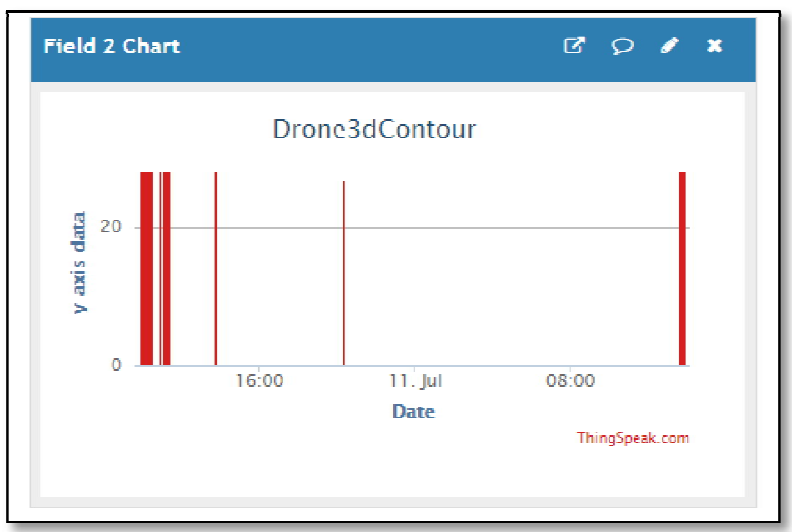

Figure 8b: Y position Database from ThingSpeak 


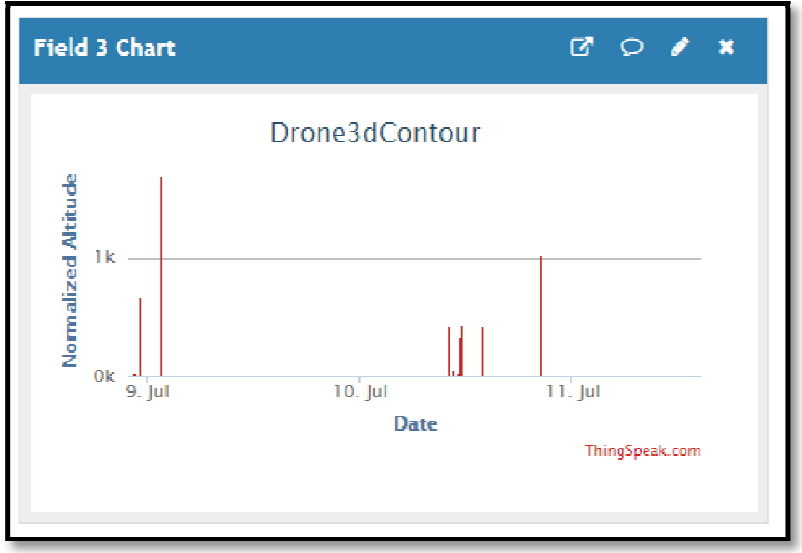

Figure 8c: Altitude Database from ThingSpeak

2D contour map (Figure 9), 3D contour map (Figure 10) and 3D model (Figure 11) that are created using MATLAB function shows the altitude information for analysis and visualization of captured information from proposed system.

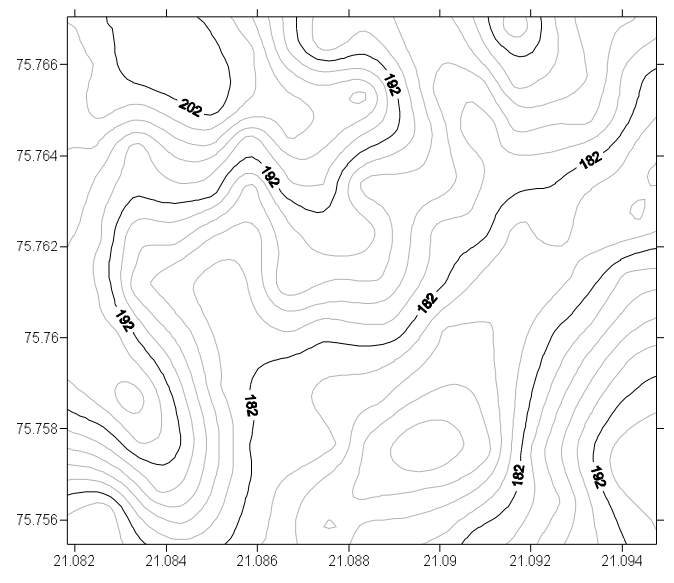

Figure 9: 2D Contour map of Surveyed Field

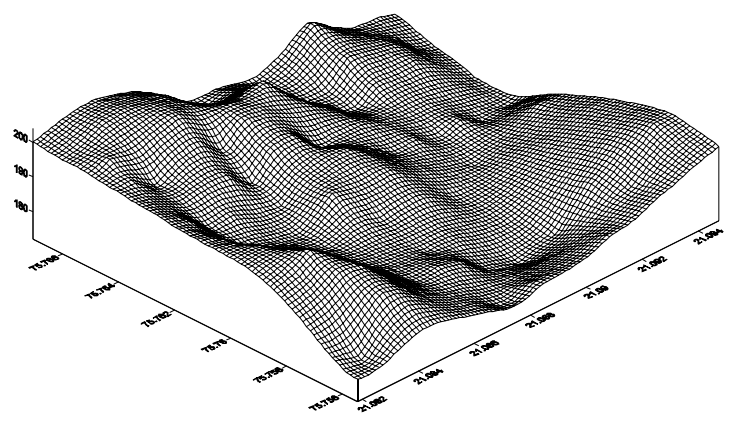

Figure 10: 3D Contour map of Surveyed Field

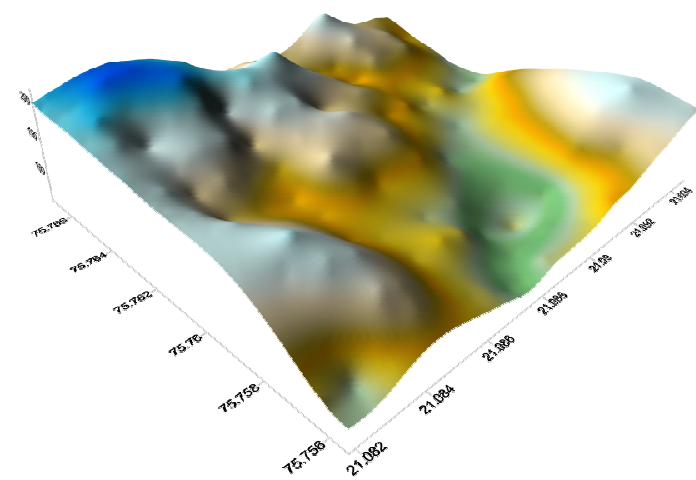

Figure 11: 3D Model of Surveyed Field

ASP.NET web-server is designed using MATLAB production server and .NET development. The various web pages are deployed into the web-server for remote access. Authentication web page (Figure 12), Data Entry web page (Figure 13) and the result page (Figure 14) is displayed for recently made survey at Anjale Ghat near Tal. Bhusawal Dist. Jalgaon in Maharashtra state of India.

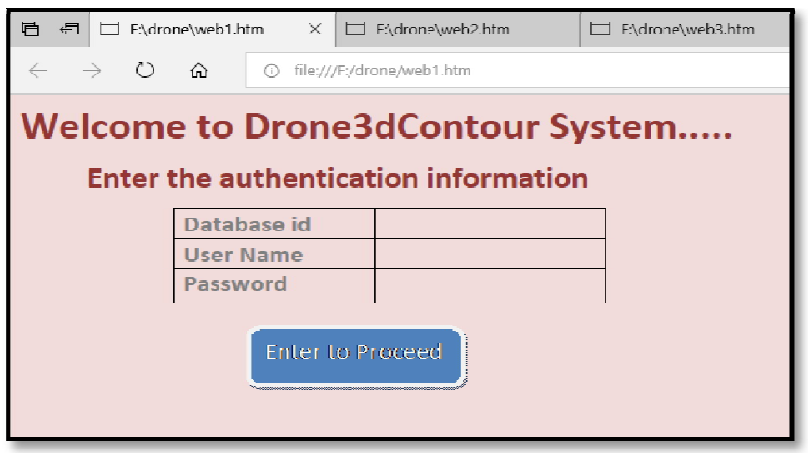

Figure 12: Authentication Web Page

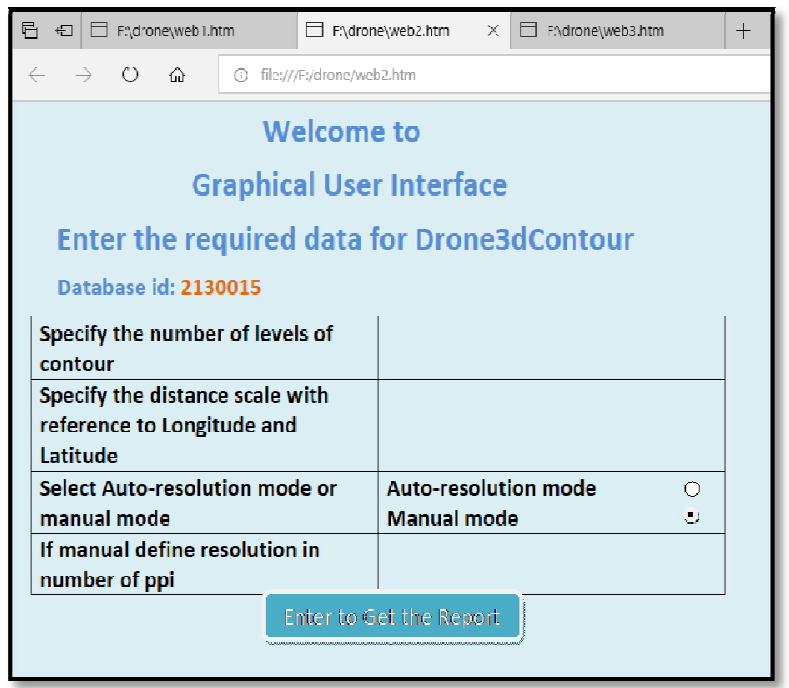

Figure 13: Data Entry Web Page

The proposed system may be integrated with Cloud based System [17] and Raspberry Pi frameworks [18, 19]. 


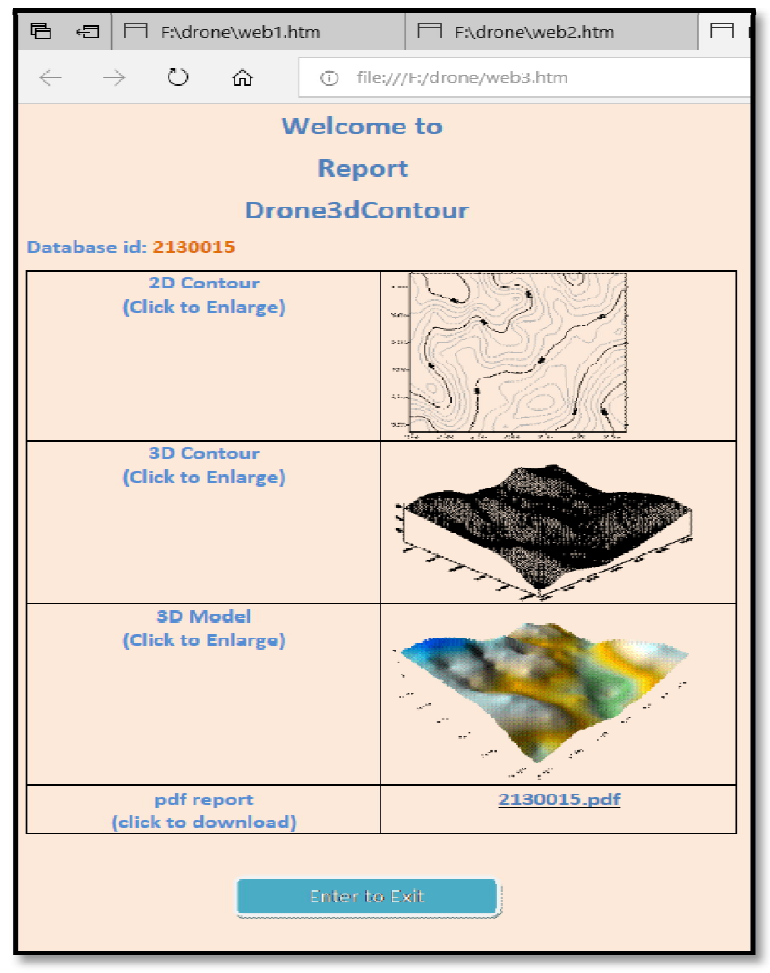

Figure 14: Result Web Pages that can be Accessed Remotely

\section{CONCLUSION}

Geographic and Cartesian data sets collection is a tedious job in contour formation that includes $\mathrm{X}, \mathrm{Y}$ information with corresponding altitude. This work demonstrates the easy, error free (accurate), quick and efficient way of geographic and Cartesian data sets creation and contour formation. Using Controlled Drone, the surveying field is scanned along pre-defined path for pre-defined point of scan to measure its altitude from earth surface in normal direction. The data is compiled and directed to cloud for storage through communication controllers like LRLDSC (for local communication) and Base Station (for global communication). Mathworks ThingSpeak cloud and MATLAB Computing function is used for storing data and for creation of 2D contour and 3D model in terms of maps. Accessibility of captured information and created maps is possible using developed website that can be deployed into .NET web-server. The required information is easily accessible remotely from any place using proposed system with proper authentication method.

\section{REFERENCES}

1. Tanaka, K., The relief contour method of representing topography on maps, Geographical Review, 40 (3), 444-456, 1950, doi:10.2307/211219.

2. http://terraincartography.com/PyramidShader/

3. https://mangomap.com/gis-software

4. McGrath, K.J., Hillshading with illuminated contours, Cartographic Perspectives, 64, 62-63, 2009, doi:10.14714/CP64.151.

5. Wessel, P., and J. F. Luis, The GMT/MATLAB Toolbox, Geochem. Geophys. Geosyst, 18, 811-823, 2017.

6. A. L. Montealegre, M. T. Lamelas and J. de la Riva, A Comparison of Open-Source LiDAR Filtering
Algorithms in a Mediterranean Forest Environment, IEEE Journal of Selected Topics in Applied Earth Observations and Remote Sensing, vol. 8, no. 8, pp. 4072-4085, Aug. 2015, doi: 10.1109/JSTARS.2015.2436974.

7. http://www.ncarg.ucar.edu/gks/gkshome.html

8. H. Shakhatreh et al., Unmanned aerial vehicles (UAVs): A survey on civil applications and key research challenges, IEEE Access, vol. 7, pp. 4857248634, 2019.

9. L. Meier, P. Tanskanen, F. Fraundorfer and M. Pollefeys, PIXHAWK: A system for autonomous flight using onboard computer vision, IEEE International Conference on Robotics and Automation, Shanghai, 2011, pp. 2992-2997, doi: 10.1109/ICRA.2011.5980229.

10. K. Sabanc1, E. Yigit, D. Üstün, A. Toktaş and Y. Çelik, Thingspeak Based Monitoring IoT System for Counting People in A Library, International Conference on Artificial Intelligence and Data Processing (IDAP), Malatya, Turkey, 2018, pp. 1-6, 2018 doi: 10.1109/IDAP.2018.8620793.

11. Kantilal Rane, Design and Development of IOT, Web-Server and ML-AVPR based Intelligent Humanoid Robot for Traffic Assistance, International Journal of Advanced Trends in Computer Science and Engineering, Vol. 9(2), PP 1922-1929, 2020.

https://doi.org/10.30534/ijatcse/2020/157922020

12. Dirk-Jan Kroon, Web Server (https://www.mathworks.com/matlabcentral/fileexc hange/29027-web-server), MATLAB Central File Exchange.

13. https://in.mathworks.com/products/matlab-compilersdk.html

14. https://in.mathworks.com/help/mps/

15. David L. Kulhavy, I-Kuai Hung, Daniel R. Unger and Yanli Zhang, Accuracy Assessment on Drone Measured Heights at Different Height Levels,(https://scholarworks.sfasu.edu/cgi/viewcontent cgi ? article $=1005 \&$ context $=$ soar .

16. https://www.dronedeploy.com/

17. Ilham Al Fajri, Oscar Tristan Sidanta, Gunawan Wang and Nilo Legowo, Design Restaurant Information System (RIS) Cloud Based with the Zachman Framework Approach, International Journal of Emerging Trends in Engineering Research, vol.8 No 5, PP. 1756 - 1760, 2020.

https://doi.org/10.30534/ijeter/2020/43852020

18. Kantilal P Rane, Online Rpi-Web-Server based Blood Cell Analysis for Fast Diagnosis and Monitoring of Disorders for Remote Stations, International Journal of Emerging Trends in Engineering Research, vol.8 No 4, PP. 1366 - 1372, 2020.

https://doi.org/10.30534/ijeter/2020/69842020

19. Ashish Patwari, Saicharan M, Sricharan L and Hemanth Penta, Microphone Array and Raspberry Pi Interfacing for Real-time DOA Estimation and Tracking of Audio Sources, International Journal of Emerging Trends in Engineering Research, vol.8 No 5, PP. 1972 - 1978, 2020.

https://doi.org/10.30534/ijeter/2020/82852020 